\title{
The Relationship of Clinical Symptoms with Social Cognition in Children Diagnosed with Attention Deficit Hyperactivity Disorder, Specific Learning Disorder or Autism Spectrum Disorder
}

\author{
Berkan Şahin $^{\circledR}$, Koray Karabekiroğlu², Abdullah Bozkurt ${ }^{3}$, Miraç Barış Usta ${ }^{4}$, \\ Muazzez Aydın², and Cansu Çobanoğlu² \\ ${ }^{1}$ Department of Child and Adolescent Psychiatry, Iğdır State Hospital, Iğdır, Turkey \\ 2Department of Child and Adolescent Psychiatry, Ondokuz Mayıs University Faculty of Medicine, Samsun, Turkey \\ ${ }^{3}$ Department of Child and Adolescent Psychiatry, Konya Training And Research Hospital, Konya, Turkey \\ ${ }^{4}$ Department of Child and Adolescent Psychiatry, Samsun Training And Research Hospital, Samsun, Turkey
}

Objective One of the areas of social cognition is Theory of Mind (ToM) is defined as the capacity to interpret, infer and explain mental states underlying the behavior of others. When social cognition studies on neurodevelopmental disorders are examined, it can be seen that this skill has not been studied sufficiently in children with Specific Learning Disorder (SLD).

Methods In this study, social cognition skills in children diagnosed with attention deficit hyperactivity disorder (ADHD), SLD or Autism Spectrum Disorder (ASD) evaluated before puberty and compared with controls. To evaluate the ToM skills, the first and secondorder false belief tasks, the Hinting Task, the Faux Pas Test and the Reading the Mind in the Eyes Task were used.

Results We found that children with neurodevelopmental disorders as ADHD, ASD, and SLD had ToM deficits independent of intelligence and language development. There was a significant correlation between social cognition deficits and problems experienced in many areas such as social communication and interaction, attention, behavior, and learning.

Conclusion Social cognition is an important area of impairment in SLD and there is a strong relationship between clinical symptoms and impaired functionality.

Psychiatry Investig 2018;15(12):1144-1153

Key Words Specific Learning Disorder, Autism, Attention deficit hyperactivity disorder, Social cognition, Theory of Mind.

\section{INTRODUCTION}

Social cognition is the social psychology area which aims to understand people's mental states including beliefs, desires, hopes, wishes, and emotions to be able to conform to changing surroundings and understand their place in the social world. ${ }^{1,2}$ One of the areas of social cognition is Theory of Mind (ToM), which is defined as the capacity to interpret, infer and explain mental states underlying the behavior of others, including the understanding of false beliefs, hints, purpose, humor, tricks, metaphor and irony. ${ }^{3}$

Received: June 24, 2018 Revised: August 4, 2018

Accepted: October 1, 2018

$\triangle$ Correspondence: Berkan Şahin, MD

Department of Child and Adolescent Psychiatry, Iğdır State Hospital, 76000, Iğdır, Turkey

Tel: +90-546-4823293, Fax: +90-476-2276358, E-mail: mail.berkan@gmail.com

(c) This is an Open Access article distributed under the terms of the Creative Commons Attribution Non-Commercial License (http://creativecommons.org/licenses/by$\mathrm{nc} / 4.0$ ) which permits unrestricted non-commercial use, distribution, and reproduction in any medium, provided the original work is properly cited.
ToM skills are necessary for a person to be able to understand that their mental state is different from the mental state of others and to be able to draw the correct conclusions from the mental state of others. Taking into account the difficulty of defending the ToM concept as a single ability, evaluations have been made of different components. First-order false belief (FOFB), Second-order false belief (SOFB), metaphor, irony and Faux Pas concepts have been identified as components related to the cognitive system. ${ }^{4}$ In addition, the Reading the Mind in the Eyes test is often used to test the ability to perceive the mental state of others based on information that can be directly observed and is related to the affective system. Cognitive social cognition skills are required when asked what someone's belief and affective social cognition skills are required when asked how someone feels. ${ }^{5}$

In the field of psychopathology, Baron-Cohen first showed in 1985 that ToM skills in autism and other common developmental disorders were an important social skill in inter- 
personal communication. And these skills were poor in children with autism. ${ }^{6}$ Following ToM studies in autistic children, further studies were conducted examining the ToM skills in groups with different diagnoses. Many social cognition studies have been conducted on groups diagnosed with attention deficit hyperactivity disorder (ADHD) in particular, of the neurodevelopmental disorders group. As these children have a reduced capacity for social reciprocity and understanding social clues, and that these features are similar to the social interaction problems of the core symptoms of Autism Spectrum Disorder (ASD). It has been emphasised that for many children with ADHD, the deficiency of giving appropriate social responses and the perception of the behavior of others is characterised by inappropriate social behavior. It has been hypothesized that the inappropriate social behavior of children diagnosed with ADHD could be phenomenologically and etiologically related to ASD. ${ }^{7}$ Although this group has been seen to have better ToM skills than ASD groups, it is thought that the low level of ToM skills could contribute to the interpersonal and behavioral problems in ADHD. ${ }^{8}$ Despite separate evaluations of ASD and ADHD groups in respect of ToM skills, there has been an insufficient examination of the neurodevelopmental disorder of Specific Learning Disorder (SLD) in social cognition studies. As this cluster of disorders is often seen in combination, this may cause difficulties in the evaluation of social cognition studies. The problems experienced in the social emotions area of children diagnosed with SLD are known to have a negative effect on self-esteem, establishing social relationships and maintaining skills and these can accompany psychiatric comorbidities. ${ }^{9}$ It is thought that the deficit in social cognition skills could be important in this process.

In the light of this knowledge, the aim of this study was to evaluate ToM skills in prepubertal children diagnosed with ADHD, ASD, and SLD, to determine whether or not there was a deficiency in ToM skills and to compare these skills between the groups and with children showing typical development. A secondary aim of the study was to investigate the relationship between impairment of the ToM skills and clinical symptoms.

\section{METHODS}

\section{Participants}

This study was designed as a single-center, cross-sectional controlled study. The patient groups were recruited from children presenting at the Child and Adolescent Mental Health and Disorders Polyclinic of Ondokuz Mayis University Medical Faculty Training and Research Hospital. The control group was recruited from volunteer relatives of health workers. All parents signed informed consent for participation in this study. The groups comprised 24 children aged 7-12 years diagnosed with ADHD, 24 children aged 7-12 years diagnosed with SLD, 26 children aged 7-12 years diag-

Table 1. Demographic and clinical characteristics of the total sample

\begin{tabular}{|c|c|c|c|c|c|c|}
\hline & SLD (N=24) & $\operatorname{ADHD}(\mathrm{N}=24)$ & $\operatorname{ASD}(\mathrm{N}=24)$ & Control $(\mathrm{N}=26)$ & Test statistic & $\mathrm{p}$ \\
\hline Gender (\%) & & & & & $\chi^{2}=5.753^{*}$ & 0.124 \\
\hline Male & $16(66.7)$ & $18(75.0)$ & $23(88.5)$ & $20(83.3)$ & & \\
\hline Female & $8(33.3)$ & $6(25.0)$ & $3(11.5)$ & $4(16.7)$ & & \\
\hline Age & $9(7-12)$ & $9(7-12)$ & $9.5(7-12)$ & $10(7-12)$ & $\chi^{2}=4.036^{\dagger}$ & 0.258 \\
\hline \multicolumn{7}{|l|}{ IQ } \\
\hline TIQ & $80.5(70-112)^{\mathrm{a}}$ & $96.5(82-128)^{\mathrm{bc}}$ & $88.5(70-103)^{\mathrm{ac}}$ & $108(80-127)^{\mathrm{b}}$ & $\chi^{2}=36.2^{*}$ & $<0.001$ \\
\hline VIQ & $79.5(67-101)^{\mathrm{a}}$ & $97.5(84-134)^{\mathrm{b}}$ & $83(63-111)^{\mathrm{a}}$ & $108(81-125)^{\mathrm{b}}$ & $\chi^{2}=39^{*}$ & $<0.001$ \\
\hline PIQ & $86(72-126)^{\mathrm{a}}$ & $91(84-122)^{\mathrm{bc}}$ & $88(64-111)^{\mathrm{ac}}$ & $108.5(82-126)^{b}$ & $\chi^{2}=24.2^{*}$ & $<0.001$ \\
\hline \multicolumn{7}{|l|}{ PPVT } \\
\hline Raw points & $74(49-92)^{\mathrm{a}}$ & $80(63-92)^{a}$ & $75(53-89)^{\mathrm{a}}$ & $87.5(68-94)^{b}$ & $\chi^{2}=29.5^{*}$ & $<0.001$ \\
\hline Language age (months) & $108(88-135)^{\mathrm{a}}$ & $116(10.3-130)^{\mathrm{ab}}$ & $111(81-150)^{\mathrm{ab}}$ & $123(102-130)^{\mathrm{b}}$ & $\chi^{2}=10.9^{*}$ & 0.012 \\
\hline \multicolumn{7}{|l|}{ Scales } \\
\hline $\mathrm{ABC}$ & $31.5(3-123)^{\mathrm{a}}$ & $38(8-101)^{\mathrm{a}}$ & $39(0-120)^{\mathrm{a}}$ & $2(0-98)^{b}$ & $\chi^{2}=27.1^{*}$ & $<0.001$ \\
\hline SRS & $66(20-122)^{\mathrm{a}}$ & $56(27-105)^{\mathrm{a}}$ & $73(32-145)^{\mathrm{a}}$ & $23(4-86)^{b}$ & $\chi^{2}=31^{*}$ & $<0.001$ \\
\hline T-DSM-IV-S & $33(5-82)^{\mathrm{a}}$ & $38(13-74)^{\mathrm{a}}$ & $22(1-84)^{\mathrm{a}}$ & $6(0-26)^{\mathrm{b}}$ & $\chi^{2}=30.7^{*}$ & $<0.001$ \\
\hline
\end{tabular}

${ }^{*}$ Kruskal Wallis test statistic, ${ }^{\dagger}$ Pearson’s chi-squared test statistic. SLD: specific learning disorder, ADHD: attention deficit hyperactivity disorder, ASD: autism spectrum disorder, a-c: no difference between groups with the same letter, IQ: intelligence quotient, PPVT: peabody picture vocabulary test, ABC: aberrant behavior checklist, SRS: social responsiveness scale, T-DSM-IV-S: Turgay DSM-IV disruptive behavior disorders rating scale 
nosed with ASD and 24 healthy children as the control group (Table 1). The diagnoses of the patient groups were made according to the DSM- 5 criteria and a score of $>70$ on the WISC-R intelligence test was included in the study. The exclusion criteria for all the patient groups had comorbid psychotic disorder, bipolar disorder, eating disorder, substance abuse, neurological disease, a history of trauma with $>1$ hour of loss of consciousness, unstable or chronic medical disease, a known sight or hearing deficit, or diagnosis of schizophrenia, intellectual disability, bipolar disorder or ASD in a parent or sibling. In the ADHD group, additional exclusion criteria were defined as taking psychiatric medication within the previous year, or an additional diagnosis of ASD or SLD. In the ASD group, an additional diagnosis of SLD, and in the SLD group an additional diagnosis of ASD were defined as exclusion criteria. The healthy control group was recruited from participants with similar characteristics in terms of age and gender averages, who attained $>70$ scores in the WISC-R test, had no history of psychiatric or medical illness, no diagnosis of ADHD, SLD, ASD, schizophrenia, intellectual disability, bipolar disorder in a parent or sibling.

\section{Procedures}

After evaluation of the diagnoses of the study groups defined for the study, the ADHD, ASD, SLD groups and the control group and their parents were given detailed information about the study. Written informed consent was obtained from the parents or legal guardians of all the participants and verbal consent was received from the children. Comorbidity was evaluated using the Schedule for Affective Disorders and Schizophrenia for School-Age Children Present Version (KSADS-P). ${ }^{10,11}$ The WISC-R was applied to all cases in our study and participated in evaluating verbal, performance and total intelligence scores.

The Childhood Autism Rating Scale (CARS) ${ }^{12,13}$ was used to determine the level of autistic symptoms, the Aberrant Behavior Checklist $(\mathrm{ABC})^{14,15}$ to evaluate irritability, agitation, crying, lethargy, social withdrawal, stereotypical behavior, hyperactivity, non-conformity and inappropriate speech, the Turgay DSM-IV Disruptive Behavior Disorders Rating ScaleTeacher form (T-DSM-IV-S) ${ }^{16}$ to assess attention, hyperactivity, impulsivity, defiance and behavioral problems, and the Social Responsiveness Scale (SRS) ${ }^{17}$ was completed by the parents to evaluate poor social reciprocity, including observable reciprocal social behavior, social use of language and pathognomic autistic behaviors. The CARS was applied to the ASD group only and all the other scales were applied to all the participants.

With the consideration that there was a relationship between language development and ToM skills performance, the Peabody Picture Vocabulary Test (PPVT) was applied to all the participants to evaluate receptive language development. ${ }^{18,19}$

We aimed to evaluate both affective and cognitive social cognition. In this direction, tests were used which were frequently used in previous studies and were validated in Turkish. To evaluate the ToM skills, the first-order false belief tasks, ${ }^{20}$ the second-order false belief tasks, ${ }^{21}$ the Hinting Task, ${ }^{22}$ the Faux Pas Test ${ }^{23}$ and the Reading the Mind in the Eyes Task (RMET) ${ }^{24}$ were used. ToM measures were all completed by participants and psychiatric scales of interference and symptomatology were all filled out by the parents.

\section{ToM tests}

To evaluate the ToM skills in the study, 2 First-order and 2 Second-order ToM tasks, the Hinting Task formed of 4 stories and the Faux Pas Test formed of a total of 10 stories, including 5 control stories, were used. These tasks consist of short stories and related questions. At the end of the stories, the children were asked questions to evaluate the ToM skills. In the REMT, photographs of the eye area of faces were shown and expected to respond correctly in understanding another person's emotions by children. The scores of correct responses to all the questions indicate the ToM skill performance.

First-order false belief tasks evaluate the first-order false belief skills and these skills that can be conceptualized at the simplest level as what one person knows and another person does not know. It is defined as the ability of one person to understand others' misconceptions, thoughts and it is the belief of a person related to their world. ${ }^{25}$ In the first-order false belief tasks, the Sally-Anne test and the Smarties test were used..$^{20}$ Smarties box was showed and asked what it contained in "Smarties test." Subsequently, they were shown that the box contained a pencil. The test question was to predict what another child, who had never seen the box, would think it contained. One point was obtained if the participant answered smarties, candy or chocolate. The card is shown to the participant, pointing to the card (Sally, Anne, a box, a basket and a ball) in "Sally-Anne test." The story was told with drawings on cards. The participants were asked where Ann had put the ball and where it really was now. A score of 1 point was given if both questions were answered correctly.

The function of the second-order false belief is the ability to predict the thoughts of a second person about the thoughts of a third person. ${ }^{26}$ The participants were requested to make a prediction taking into account the information the person in the story had about a third person. In the secondorder false belief tests, the stories for the Chocolate Bar Task and the Ice-Cream Truck Task were used. ${ }^{21}$ The false belief tasks have been translated into Turkish and reliability studies 
have been conducted..$^{27}$ The participants listened the stories about two children through the drawings on both tests. One of the stories was about the ice cream truck, another chocolate box. After listening the story, participants were asked to predict one's belief about other's belief. Two test were scored in a pass/fail manner. If the participant made no mental state attributions, received a score of 0 . If mental states were attributed for two characters, a score of 1 was given. Total False Belief Level (TFBL), a composite ToM performance score, was generated by summing both orders of false belief tasks and it was compared between the groups.

The Hinting Task is one of the advanced level ToM functions. ${ }^{28}$ It tests the skill of being able to predict the real intention behind the words directly spoken. After listening the story, the participants were asked what the person in the story really had wanted to say. If the participant did not respond correctly to the first hint question, the practitioner moved on to a question including a clearer hint. If the correct answer was given for the first hint, a score of 2 was given, if the answer with a clearer hint was true, a score of 1 was given. If both hint questions were not answered correctly, a score of 0 was given. In the current study, 4 stories were used in the Hinting Task. ${ }^{22}$

The Faux Pas Test was used to evaluate high mental crossreferencing. ${ }^{23}$ Noticing a faux pas is accepted as the most complex skill developmentally and it is accepted as a sensitive measurement tool for ToM. A faux pas occurs when a person says something that they should not have, without knowing or realizing. To be able to understand when a faux pas has been made, it is necessary to represent two mental states. This skill requires both concept skill elements and emotional empathy elements. ${ }^{25,26}$ After listening the story, four questions were asked to assess the child's understanding. To detect a faux pas the child has to answer all the questions correctly, answer a comprehension question, and recognize that the faux pas was a consequence of a false belief. In control stories, child has to detect that no faux pas took place. Failure of any of these questions leads to a score of zero for that story. The children's version of the Faux Pas Recognition Test, which was developed by Baron-Cohen, was applied in Turkish with 5 original faux pas stories and 5 control stories (maximum score was 10 points). ${ }^{23}$

The Reading the Mind in the Eyes Task (RMET) was developed by Baron-Cohen et al. ${ }^{29}$ as an advanced ToM test. In 2001, it was revised for use with children, using the photographs of the eyes of 28 females and males. With the inclusion of functions such as facial perception and recognizing emotions, it aims to test to what extent the participants can put themselves in another's place and to what extent they can conceptualize their mental state. ${ }^{24}$ After each picture of the eye was shown, asked to choose the best one of 4 options. A score of 1 was given for each correct answer (maximum score was 28 points). The test was validated in Turkish. ${ }^{30}$

\section{Statistical analysis}

The data were analyzed using IBM SPSS Statistics version 23.0 software (IBM Corp., Armonk, NY, USA). The conformity to the normal distribution was tested with the Shapiro Wilk test. Quantitative data with normal distribution were compared between the groups using one-way variance analysis (ANOVA) and the ANCOVA test. Data not showing normal distribution were compared using the non-parametric Kruskal Wallis test. To determine from which group any significant difference originated, the Mann Whitney U-test with Bonferroni correction was applied. The relationships between categorical variables were examined with the chi-square test. In the examination of relationships between quantitative data, Spearman correlation analysis was used. A value of $\mathrm{p}<0.05$ was accepted as statistically significant.

\section{Ethics board approval}

Approval for the study was granted by the Clinical Research Ethics Committee of Ondokuz Mayis University (B.30. ODM.0.20.08/632-745).

\section{RESULTS}

\section{Demographic and clinical characteristics of the subjects}

From the children initially invited into the study groups, 8 were excluded with a diagnosis of intellectual disability, 2 with a diagnosis of epilepsy, 2 with psychiatric disorders diagnosed in first-degree relatives and 2 as they did not wish to continue with the tests. The patient and control groups were formed taking into consideration the age, gender, and education level of the children, with a total of 94 included for evaluation. Diagnoses were made according to the DSM-5 criteria. The ADHD group comprised 24 children, the SLD group comprised 24 children, the ASD group comprised 26 children and the control group was formed of 24 children with no psychiatric diagnosis. The children comprised 77 (78.6\%) males and $21(21.4 \%)$ females and all were in the age range of 7-12 years. The distributions of mean age and gender were found to be similar between the groups (Table 1).

The WISC-R verbal, performance and total scores of all the participants were evaluated. The language development PPVT raw scores and the socioculturally corrected Receptive Language Age (months) were compared between the groups. A difference was determined between the groups in respect of the intelligence level and the receptive language level (Table 1). There was no difference between the control and ADHD 
groups. In respect of the total intelligence level, the ASD and SLD groups were determined to have statistically significantly lower scores. The verbal IQ scores were higher than performance IQ in the ADHD group and the performance IQ scores were higher than verbal IQ in the ASD and SLD groups. When the receptive language level evaluated with the PPVT was examined, all 3 patient groups had significantly low raw scores, and when the corrected language age was examined, only the SLD group was determined to have a significantly lower language age than the control group.

\section{Evaluation of the scale and test scores}

The children in the study were evaluated with the KSADS-P-T to determine comorbidity. Comorbidity was found as ADHD (62.5\%) and Oppositional Defiant Disorder (ODD) (58.3\%) in the SLD group, as ODD (70.9\%) and Specific Phobia (29.2\%) in the ADHD group and as ADHD (80.8\%) and Specific Phobia (34.6\%) in the ASD group. No significant difference was determined between the groups in respect to the rates of comorbidities.

The scores of the ABC, SRS and the T-DSM-IV-S/Family Form applied to all the participants were compared between the groups and the results are shown in Table 1. When the groups were compared in respect of the total scores of all 3 scales, no significant difference was determined between the patient groups, and statistically, significantly higher scores were determined in the patient groups compared to the control group $(\mathrm{p}<0.001)$.

\section{Evaluation of the ToM tasks}

The ToM tasks results of all the groups are shown in Table 2. A statistically significant difference was determined between the groups. When the success rates of the Smarties Test and the Ice-Cream Truck Test were examined, a significantly low rate was only seen in the ASD group compared to controls. When all the false belief tests were evaluated, all 3 patient groups were observed to have shown a low performance. In the Hinting tasks, the Faux Pas Test and the REMT, low scores were obtained at a similar level in all 3 patient groups, with no significant difference between them, and these were all statistically significantly lower than the scores of the control group $(\mathrm{p}<0.001)$. As expected, the lowest ToM task scores were determined in the ASD group (Table 2). The correlations between the ToM skills and intelligence, receiver language age, scale scores were examined and shown in Table 3.

\section{Advanced analysis of the ToM performances}

It has been shown in previous studies that ToM development and ToM skills are influenced by many factors. In neurodevelopmental disorders, it is difficult to create pure groups in the absence of comorbidities and in terms of cofactors. Moreover, this selection may limit the clinical assessment of the nature of the disease. For this reason, we want to evaluate these cofactors by conducting advanced analysis in our present study.

In the examination of the presentation of ADHD in the ADHD group, 14 (58.3\%) cases were seen to have attention deficit and $10(41.7 \%)$ had a combined presentation. No significant difference was determined between these presentation groups in respect of ToM performance ( $p>0.05)$. In the ADHD group, a high rate of comorbidity was found such as ODD at $70.9 \%$. It was thought that this high rate of comorbidity could have been a confounding factor affecting the ToM performance in the ADHD group. Therefore, the ADHD group were separated into those with and without an additional diagnosis of ODD and the ToM performance val-

Table 2. Evaluation of the ToM tasks

\begin{tabular}{|c|c|c|c|c|c|c|c|}
\hline ToM tasks & & $\operatorname{SLD}(\mathrm{N}=24)$ & $\operatorname{ADHD}(\mathrm{N}=24)$ & $\operatorname{ASD}(\mathrm{N}=24)$ & Control $(\mathrm{N}=26)$ & Test statistic & $\mathrm{p}$ \\
\hline Smarties & success N (\%) & $22(91.7)^{\mathrm{a}}$ & $22(91.7)^{\mathrm{a}}$ & $15(57.7)^{\mathrm{b}}$ & $24(100)^{\mathrm{a}}$ & $\chi^{2}=20.7^{*}$ & $<0.001$ \\
\hline Sally-Anne & success N (\%) & $14(58.3)^{\mathrm{a}}$ & $11(45.8)^{\mathrm{ab}}$ & $8(30.8)^{b}$ & $24(100)^{\mathrm{c}}$ & $\chi^{2}=26.7^{*}$ & $<0.001$ \\
\hline Chocolate Bar & success N (\%) & $11(45.8)^{\mathrm{a}}$ & $8(33.3)^{\mathrm{a}}$ & $8(30.8)^{\mathrm{a}}$ & $19(79.2)^{\mathrm{b}}$ & $\chi^{2}=14.5^{*}$ & $<0.001$ \\
\hline Ice-Cream Truck & success N (\%) & $9(37.5)^{\mathrm{ab}}$ & $7(29.2)^{\mathrm{ab}}$ & $4(15.4)^{\mathrm{b}}$ & $15(62.5)^{\mathrm{a}}$ & $\chi^{2}=12.6^{*}$ & 0.005 \\
\hline FOFB & Md (range) & $2(0-2)^{c}$ & $1(0-2)^{\mathrm{cb}}$ & $1(0-2)^{b}$ & $2(2-2)^{a}$ & $\chi^{2}=31^{\dagger}$ & $<0.001$ \\
\hline SOFB & Md (range) & $1(0-2)^{\mathrm{ab}}$ & $0.5(0-2)^{\mathrm{b}}$ & $0(0-2)^{b}$ & $2(0-2)^{a}$ & $\chi^{2}=16.9^{\dagger}$ & 0.001 \\
\hline TFBL & Md (range) & $2(0-4)^{a}$ & $2(0-4)^{\mathrm{a}}$ & $1(0-4)^{\mathrm{a}}$ & $4(2-4)^{\mathrm{b}}$ & $\chi^{2}=30^{\dagger}$ & $<0.001$ \\
\hline Hinting task & Md (range) & $3(0-7)^{\mathrm{a}}$ & $4(0-8)^{\mathrm{a}}$ & $2(0-6)^{\mathrm{a}}$ & $8(1-8)^{b}$ & $\chi^{2}=44.8^{\dagger}$ & $<0.001$ \\
\hline Faux pas test & Md (range) & $3.5(0-8)^{\mathrm{a}}$ & $4.5(1-7)^{\mathrm{a}}$ & $1.5(0-7)^{\mathrm{a}}$ & $8(4-10)^{b}$ & $\chi^{2}=50.2^{\dagger}$ & $<0.001$ \\
\hline RMET & $\mathrm{M} \pm \mathrm{SD}$ & $12.4 \pm 5.4^{\mathrm{a}}$ & $14.9 \pm 5.3^{\mathrm{a}}$ & $12.1 \pm 5.6^{\mathrm{a}}$ & $19.5 \pm 3.6^{\mathrm{b}}$ & $\mathrm{F}=11.0^{\ddagger}$ & $<0.001$ \\
\hline
\end{tabular}

*Pearson’s chi-squared test statistic, ${ }^{\dagger}$ Kruskal Wallis test statistic, ${ }^{\ddagger}$ one-way ANOVA. SLD: specific learning disorder, ADHD: attention deficit hyperactivity disorder, ASD: autism spectrum disorder, FOFB: first-order false belief, SOFB: second-order false belief, TFBL: total false belief level, RMET: reading the mind in the eyes task, a-c: no difference between groups with the same letter 
ues were compared again with the control group and re-analyzed (Table 4).

In the SLD group, when the types of SLD were examined, reading, writing, and arithmetic learning disorder types were seen most frequently ( $\mathrm{n}: 14,58.3 \%)$. No difference was determined between the types of SLD in respect of ToM performance ( $p>0.05)$. The SLD group had a high rate of comorbid ADHD as $62.5 \%$. It was thought that this high rate of comorbity could have been a confounding factor affecting the ToM performance in the SLD group. Therefore, the ADHD group, the SLD group without $\mathrm{ADHD}(\mathrm{n}: 9)$ and the control group were re-analyzed in respect of ToM performance values (Table 5).

Advanced examinations were applied because of the difference between the participants in respect of intelligence and receptive language levels. A statistically significant positive correlation was found between all the ToM skills and intelligence and receptive language age $(\mathrm{p}<0.05, \mathrm{r}: 0.27-0.68)$. Therefore, these two development levels, which could be potential confounding

Table 3. Correlations between the ToM skills and intelligence, receiver language age, scale scores

\begin{tabular}{lcccccrrr}
\hline & & FOFB & SOFB & TFBL & Hinting task & Faux pas test & RMET \\
\hline RLA & $\mathrm{r}$ & 0.380 & 0.235 & 0.342 & 0.434 & 0.441 & 0.505 \\
TIQ & $\mathrm{p}$ & $<0.001$ & 0.035 & 0.002 & $<0.001$ & $<0.001$ & $<0.001$ \\
& $\mathrm{r}$ & 0.272 & 0.313 & 0.335 & 0.487 & 0.465 & 0.356 \\
SRS & $\mathrm{p}$ & 0.013 & 0.004 & 0.002 & $<0.001$ & $<0.001$ & 0.001 & -0.294 \\
& $\mathrm{r}$ & -0.326 & -0.305 & -0.359 & -0.351 & -0.426 & 0.001 & 0.008 \\
ABC & $\mathrm{p}$ & 0.003 & 0.006 & 0.001 & 0.001 & -0.358 & -0.363 & -0.222 \\
& $\mathrm{r}$ & -0.284 & -0.263 & -0.303 & 0.002 & 0.001 & 0.055 \\
T-DSM-IV-S & $\mathrm{p}$ & 0.014 & 0.023 & 0.008 & -0.250 & -0.302 & -0.231 \\
& $\mathrm{r}$ & -0.403 & -0.325 & -0.405 & 0.024 & 0.006 & 0.038 \\
CARS* & $\mathrm{p}$ & $<0.001$ & 0.003 & $<0.001$ & -0.118 & 0.177 & 0.16 \\
& $\mathrm{r}$ & -0.445 & -0.358 & -0.444 & 0.592 & 0.418 & 0.466 \\
\hline
\end{tabular}

${ }^{*}$ CARS applied only to ASD group. RLA: receiver language age, r: Spearman Correlation Coefficient. FOFB: first-order false belief, SOFB: second-order false belief, TFBL: total false belief level, RMET: reading the mind in the eyes task, TIQ: total intelligence quotient, SRS: social responsiveness scale, ABC: aberrant behavior checklist, T-DSM-IV-S: Turgay DSM-IV disruptive behavior disorders rating scale, CARS: childhood autism rating scale

Table 4. Evaluation the impact of ODD on ToM performance in ADHD group

\begin{tabular}{lcccccc}
\hline & FOFB & SOFB & TFBL & Hinting task & Faux pas test & RMET \\
\hline ADHD+ODD & $2(0-2)^{\mathrm{ab}}$ & $0(0-2)^{\mathrm{a}}$ & $2(0-4)^{\mathrm{a}}$ & $14(8-22)^{\mathrm{a}}$ & $4(1-6)^{\mathrm{a}}$ & $14(8-22)^{\mathrm{a}}$ \\
ADHD & $1(1-2)^{\mathrm{a}}$ & $1(0-2)^{\mathrm{a}}$ & $2(1-4)^{\mathrm{a}}$ & $17(2-22)^{\mathrm{a}}$ & $5(2-7)^{\mathrm{a}}$ & $17(2-22)^{\mathrm{ab}}$ \\
Control & $2(2-2)^{\mathrm{b}}$ & $2(0-2)^{\mathrm{b}}$ & $4(2-4)^{\mathrm{b}}$ & $19.5(10-25)^{\mathrm{b}}$ & $8(4-10)^{\mathrm{b}}$ & $19.5(10-25)^{\mathrm{b}}$ \\
Test statistic & $\chi^{2}=19.9$ & $\chi^{2}=10.1$ & $\chi^{2}=15.6$ & $\chi^{2}=21.3$ & $\chi^{2}=28.9$ & $\chi^{2}=9.6$ \\
$\mathrm{p}$ & $<0.001$ & 0.006 & $<0.001$ & $<0.001$ & $<0.001$ & 0.008 \\
\hline
\end{tabular}

a-b: no difference between groups with the same letter, $\chi^{2}$ : Kruskal Wallis test statistic. FOFB: first-order false belief, SOFB: second-order false belief, TFBL: total false belief level, RMET: reading the mind in the eyes task, ADHD: attention deficit hyperactivity disorder, ODD: oppositional defiant disorder

Table 5. Evaluation the impact of ADHD on ToM performance in SLD group

\begin{tabular}{lcccccc}
\hline & FOFB & SOFB & TFBL & Hinting task & Faux pas test & RMET \\
\hline SLD & $2(1-2)^{\mathrm{ab}}$ & $1(0-2)^{\mathrm{ab}}$ & $3(1-4)^{\mathrm{ab}}$ & $3(0-7)^{\mathrm{a}}$ & $3(0-8)^{\mathrm{a}}$ & $11(6-22)^{\mathrm{a}}$ \\
ADHD & $1(0-2)^{\mathrm{a}}$ & $0.5(0-2)^{\mathrm{a}}$ & $2(0-4)^{\mathrm{a}}$ & $4(0-4)^{\mathrm{a}}$ & $4.5(1-7)^{\mathrm{a}}$ & $15.5(2-22)^{\mathrm{a}}$ \\
Control & $2(2-2)^{\mathrm{b}}$ & $2(0-2)^{\mathrm{b}}$ & $4(2-4)^{\mathrm{b}}$ & $8(1-8)^{\mathrm{b}}$ & $8(4-10)^{\mathrm{b}}$ & $19.5(10-25)^{\mathrm{b}}$ \\
Test statistic & $\chi^{2}=19.2$ & $\chi^{2}=9.5$ & $\chi^{2}=16.2$ & $\chi^{2}=24.3$ & $\chi^{2}=32.2$ & $\chi^{2}=17.2$ \\
p & $<0.001$ & 0.009 & $<0.001$ & $<0.001$ & $<0.001$ & $<0.001$ \\
\hline
\end{tabular}

a-b: no difference between groups with the same letter, $\chi^{2}$ : Kruskal Wallis test statistic. FOFB: first-order false belief, SOFB: second-order false belief, TFBL: total false belief level, RMET: reading the mind in the eyes task, SLD: specific learning disabilities, ADHD: attention deficit hyperactivity disorder 
factors were checked separately to examine whether or not the difference between the groups existed (Table 6 and 7).

\section{DISCUSSION}

To the best of our knowledge, this is the first study to have compared social cognition skills in children diagnosed with ADHD, SLD, and ASD. ToM studies in mid-childhood are seen as a noticeable gap in the literature. Studies of schoolage children constitute only $4 \%$ of the total ToM studies. ${ }^{31}$ From both a neurological and a social perspective, direct comparisons between the pre-school period and adolescence are problematic. Therefore, by including children aged 7-12 years in this study, it was aimed to make a contribution to filling this gap in the literature.

It was aimed to evaluate the social impairment in different areas by applying scales, focused on aberrant and disruptive behaviors, social reciprocity. When the scores of the T-DSMIV-S-Family, ABC, and SRS that were used in this study were examined, it was seen that all 3 patient groups had significantly higher scores than the control group, but no significant difference was found between the patient groups. As the SLD and ASD groups had comorbid ADHD at a high rate and when the functional problems in neurodevelopmental diseases were taken into consideration, the results of disruptive and abnormal behavior were found to be high, as ex- pected. The high scores in the SRS indicated a severe impairment in social reciprocity. The scale results showed that there was social impairment in all 3 patient groups of neurodevelopmental disorders, that was not specific to the ASD group and as expected, that was most commonly seen in the ASD, SLD, and ADHD groups respectively.

ToM tasks were applied to all the children in the study. The first-order false belief performance scores of all 3 patient groups were found to be lower than those of the control group. In the second-order false belief performance, a significantly lower score was found in the ADHD and ASD groups compared to the control group. When the total scores of the false belief tasks were examined, a significantly low performance was found in all 3 patient groups compared to the control group (ASD $<\mathrm{ADHD}<\mathrm{SLD}$ ). In the advanced ToM tasks, the Hinting Task, the Faux Pas Recognition Test and the RMET, significantly low performance was found in all 3 patient groups compared to the control group but no significant difference was found between the patient groups ( $\mathrm{ASD}<\mathrm{SLD}<\mathrm{ADHD})$.

Previous social cognition studies conducted on children diagnosed with ADHD and ASD have yielded conflicting results. ${ }^{32-36}$ In a study by Dyck et al. ${ }^{37}$ in which children with ADHD, ASD, and other disorders were compared with healthy children, it was reported that ToM deficit was not specific to ASD and empathy was not independent of intelli-

Table 6. Evaluation of ToM tasks after controlling intelligence

\begin{tabular}{lcccrcc}
\hline & SLD & ADHD & ASD & Control & F & p \\
\hline FOFB & $1.591 \pm 0.59^{\mathrm{ac}}$ & $1.375 \pm 0.576^{\mathrm{ba}}$ & $0.944 \pm 0.802^{\mathrm{b}}$ & $2 \pm 0^{\mathrm{c}}$ & 8.003 & $<0.001$ \\
SOFB & $0.818 \pm 0.907$ & $0.667 \pm 0.761$ & $0.389 \pm 0.608$ & $1.389 \pm 0.778$ & 3.229 & 0.050 \\
TFBL & $2.409 \pm 1.221^{\mathrm{ac}}$ & $2.042 \pm 1.16^{\mathrm{ab}}$ & $1.333 \pm 1.138^{\mathrm{b}}$ & $3.389 \pm 0.778^{\mathrm{c}}$ & 7.605 & $<0.001$ \\
Hinting task & $2.955 \pm 2.081^{\mathrm{a}}$ & $4.25 \pm 2.212^{\mathrm{a}}$ & $2.333 \pm 1.94^{\mathrm{a}}$ & $7 \pm 1.749^{\mathrm{b}}$ & 8.547 & $<0.001$ \\
Faux pas test & $3.545 \pm 2.502^{\mathrm{a}}$ & $4.542 \pm 1.668^{\mathrm{a}}$ & $2.556 \pm 2.281^{\mathrm{a}}$ & $7.833 \pm 0.985^{\mathrm{b}}$ & 13.14 & $<0.001$ \\
RMET & $12.545 \pm 5.414$ & $14.917 \pm 5.283$ & $13.833 \pm 4.997$ & $19.444 \pm 3.434$ & 2.628 & 0.056 \\
\hline
\end{tabular}

a-c: no difference between groups with the same letter, F: One-way ANOVA. SLD: specific learning disorder, ADHD: attention deficit hyperactivity disorder, ASD: autism spectrum disorder, FOFB: first-order false belief, SOFB: second-order false belief, TFBL: total false belief level, RMET: reading the mind in the eyes task

Table 7. Evaluation of ToM tasks after controlling receiver language

\begin{tabular}{lcccccc}
\hline & SLD & ADHD & ASD & Control & F & p \\
\hline FOFB & $1.455 \pm 0.671^{\mathrm{a}}$ & $1.364 \pm 0.581^{\mathrm{bc}}$ & $0.84 \pm 0.8^{\mathrm{c}}$ & $2 \pm 0^{\mathrm{ab}}$ & 8.413 & $<0.001$ \\
SOFB & $0.818 \pm 0.853$ & $0.591 \pm 0.734$ & $0.44 \pm 0.712$ & $1.25 \pm 0.754$ & 3.726 & 0.050 \\
TFBL & $2.273 \pm 1.202^{\mathrm{ac}}$ & $1.955 \pm 1.133^{\mathrm{b}}$ & $1.28 \pm 1.308^{\mathrm{b}}$ & $3.25 \pm 0.754^{\mathrm{c}}$ & 8.156 & $<0.001$ \\
Hinting task & $2.864 \pm 2.122^{\mathrm{a}}$ & $3.955 \pm 2.058^{\mathrm{a}}$ & $2.28 \pm 2.151^{\mathrm{a}}$ & $7.083 \pm 0.996^{\mathrm{b}}$ & 12.279 & $<0.001$ \\
Faux pas test & $3.5 \pm 2.521^{\mathrm{a}}$ & $4.455 \pm 1.654^{\mathrm{a}}$ & $2.32 \pm 2.462^{\mathrm{a}}$ & $7.75 \pm 1.545^{\mathrm{b}}$ & 13.384 & $<0.001$ \\
RMET & $11.773 \pm 5.108$ & $14.682 \pm 5.286$ & $11.84 \pm 5.528$ & $18 \pm 3.977$ & 1.812 & 0.152 \\
\hline
\end{tabular}

a-c: no difference between groups with the same letter, F: One-way ANOVA. SLD: specific learning disorder, ADHD: attention deficit hyperactivity disorder, ASD: autism spectrum disorder, FOFB: first-order false belief, SOFB: second-order false belief, TFBL: total false belief level, RMET: reading the mind in the eyes task 
gence. Sinzig et al. ${ }^{38}$ examined the test results of facial emotion recognition in $\mathrm{ADHD}, \mathrm{ASD}, \mathrm{ADHD}+\mathrm{ASD}$ and control groups, and reported that the ADHD group showed a significantly worse performance than the control group and the presence of ADHD symptoms decreased ToM performance in both the ADHD only group and in the autism group. Miranda et al. examined ToM and executive functions in groups formed of children with high-functioning autism (HFA), $\mathrm{ADHD}$ and a control group. Both patient groups demonstrated low performance and the HFA group was determined to have significantly low ToM performance compared to the control group. ${ }^{39}$ Similarly, the results of the current study demonstrated that although there was lower ToM performance in the ASD group, there was no statistically significant difference between the ASD and ADHD groups.

There are very few ToM studies in the literature related to the group of learning disorders. Researchers have defined dyslexics as individuals those who tend to be disadvantaged in misperceive the social context, misjudge, and misreading social events. Patients with dyslexia have been seen to obtain lower communication skills scores than a control group in the areas of starting appropriate communication and understanding social clues. ${ }^{40}$ It is thought that children with dyslexia have emotional and social problems and have skill deficits in these areas. ${ }^{41}$ Pragmatic language skills and ToM performances were examined by Ramon et al. in a comparison of children diagnosed with dyslexia or non-verbal learning disorder and a control group. The dyslexic group was determined to have a significantly lower performance of pragmatic language and ToM skills than the control group but not to a level of statistical significance. ${ }^{42}$ Unlike the results in the literature, the SLD group in the current study demonstrated significantly lower ToM performance than the control group, and no difference was determined between the ADHD and ASD groups in respect of ToM performance.

When the relationships were examined between the ToM performances and the scale scores of the children, there was determined to be a significant negative correlation between the scale scores and the ToM skills. The high SRS scores and the low ToM performance of all 3 patient groups suggest that ToM skills in all three neurodevelopmental disorders are effective and important skills in social communication and interaction problems. In the CARS scale applied to the ASD group to evaluate the severity of autism symptoms, a moderate negative correlation was seen between the CARS scores and the first-order false beliefs performance. Although the severity of autism was an effective parameter on simple ToM skills, no significant relationship was found on advanced ToM skills. This result of the current study was similar to the findings of a study by Buitelaar et al., ${ }^{43}$ in that the presence of a diagnosis of ASD had a negative effect on advanced ToM skills independently of the severity of the disease.

It has been reported in the literature that the executive function problems in the ADHD group with a comorbid ODD, caused ToM deficits and there was a reciprocal relationship between the two skills. ${ }^{25}$ In the current study, in the comparison between the ADHD groups with and without ODD comorbidity, no significant difference was found in respect of all the ToM tasks. When the control group and the ADHD groups were compared, it was found that the RMET performances between the control group and the ADHD group without ODD were not significant. Thus, the results of the current study were similar to the findings of previous studies that have shown that ADHD with ODD had no significant effect on ToM. ${ }^{44}$ When the effect on ToM skills of comorbid ADHD diagnosis was examined in the SLD group, ADHD comorbidity in the SLD affected the simple ToM skills but there was no effect on advanced ToM performances.

Advanced analyses of intelligence and receptive language performance were made in our study. When the total intelligence level of all the participants was examined, the difference in respect of RMET performance was seen to disappear at the limit. This result was found to be consistent with a previous study that reported that RMET was predictive of the IQ scores. ${ }^{45}$ The intelligence performance affected the simple ToM skills in the SLD group. When the receptive language performance was controlled, it was found that the receptive language performance had a significant effect on RMET in all three groups of patients, while only affecting the simple ToM skills in the SLD group. The results of our study revealed a deficit in ToM skills in neurodevelopmental disorders, independently of intelligence and language development.

\section{Limitations}

Matching genders and ages among the groups will make the groups more homogenous. Examination of components related to executive functions and language (syntax, morphology, phonology, semantics, pragmatics) could have shown the effects of these on social cognition more clearly. As there could have been the poor homogeneity of the groups with comorbid diagnoses, checking all the comorbidity of the patient groups would provide clearer results of the social cognition evaluation. While the ADHD group were not using any medication, the medication status of the other groups was not checked. The use of psychiatric medication in the SLD and ASD groups could have had an effect as a confounding factor on the social cognition and neurocognitive tests. Ensuring medication control in all the groups would increase the power of future studies to be conducted in this area. As there were few patients in the current study 
who had special education intervention, the contribution of special education was not included in the analyses. Further research with a larger sample to show the effect of special education intervention on social cognition would be beneficial in respect of guiding treatment goals.

\section{Conclusion}

The data obtained at the end of this study showed that children with neurodevelopmental disorders had ToM skill deficits independently of intelligence and language development, and there was a significant correlation between these deficits and problems experienced in many areas such as social communication and interaction, attention, behavior, and learning. This suggests that social cognition is an important area of impairment in SLD and there is a strong relationship between clinical symptoms and impaired functionality. Future studies will be able to more clearly reveal the mechanism of this deficit by showing the possible common neuroanatomic structures in neurodevelopmental disorders. It can be predicted that education in ToM impairments could complement treatment planned for neurodevelopmental disorders, particularly in the treatment of difficulties experienced in social functionality. Understanding the contribution of this education to the effectiveness of treatment appears to be important. Studies based on early intervention for ToM skills, which are longitudinal and cover the period of early childhood will shed light on the development of new treatment strategies.

\section{REFERENCES}

1. Augoustinos M, Walker I, Donaghue N. Social Cognition: An Integrated Introduction. CA: SAGE Publications; 2014.

2. Wellman HM, Lagattuta KH. Theory of mind for learning and teaching: the nature and role of explanation. Cogn Dev 2004;19:479-497.

3. Yildırım E, Alptekin K. A new featured dimension in schizophrenia: social cognition. Dusunen Adam. J Psychiatr Neurol Sci 2012;25:368-375.

4. Bach LJ, Happe F, Fleminger S, Powell J. Theory of mind: independence of executive function and the role of the frontal cortex in acquired brain injury. Cogn Neuropsychiatry 2000;5:175-192.

5. Tager-Flusberg H, Sullivan K. A componential view of theory of mind: evidence from Williams syndrome. Cognition 2000;76:59-90.

6. Baron-Cohen S, Leslie AM, Frith U. Does the autistic child have a "theory of mind"? Cognition 1985;21:37-46.

7. Nijmeijer JS, Minderaa RB, Buitelaar JK, Mulligan A, Hartman CA, Hoekstra PJ. Attention-deficit/hyperactivity disorder and social dysfunctioning. Clin Psychol Rev 2008;28:692-708.

8. Bora E, Pantelis C. Meta-analysis of social cognition in attention-deficit/hyperactivity disorder (ADHD): comparison with healthy controls and autistic spectrum disorder. Psychol Med 2016;46:699-716.

9. Kavale KA, Mostert MP. Social skills interventions for individuals with learning disabilities. Learn Disabil Q 2004;27:31-43.

10. Gökler B, Ünal F, Pehlivantürk B, Kültür EÇ, Akdemir D, Taner Y. Reliability and validity of schedule for affective disorders and schizophrenia for school age children-present and lifetime version-turkish version ( $\mathrm{k}$ sads-pl-t). Turk J Child Adolesc Ment Health 2004;11:109-116.
11. Kaufman J, Birmaher B, Brent D, Rao U, Flynn C, Moreci P, et al. Schedule for affective disorders and schizophrenia for school-age children-present and lifetime version (K-SADS-PL): initial reliability and validity data. J Am Acad Child Adolesc Psychiatry 1997;36:980-988.

12. Schopler E, Reichler RJ, DeVellis RF, Daly K. Toward objective classification of childhood autism: Childhood Autism Rating Scale (CARS). J Autism Dev Disorder 1980;10:91-103.

13. Gassaloğlu SI, Baykara B, Avcil S, Demiral Y. Validity and reliability analysis of Turkish version of childhood autism rating scale. Turk Psikiyatri Derg 2016;27:266-274.

14. Aman MG, Singh N, Turbott S. Reliability of the Aberrant Behavior Checklist and the effect of variations in instructions. Am J Ment Defic 1987;92:237-240.

15. Sucuoğlu B. The psychometric characteristics of the Turkish form of the aberrant behavior checklist. Turk Psikol Derg 2003;18:77-79.

16. Turgay A. Disruptive Behavior Disorders Child and Adolescent Screening and Rating Scales for Children, Adolescents, Parents and Teachers. West Bloomfield, Michigan: Integrative Therapy Institute Publication; 1994.

17. Constantino JN, Todd RD. Intergenerational transmission of subthreshold autistic traits in the general population. Biol Psychiatry 2005;57:655-660.

18. Dunn LM, Dunn LM, Bulheller S, Häcker H. Peabody Picture Vocabulary Test. Circle Pines, Minnesota: American Guidance Service; 1965.

19. Katz JA. Turkish Peabody Picture Vocabulary Test. Hacett B Soc Sci Hum 1974;6:129-142.

20. Wimmer H, Perner J. Beliefs about beliefs: Representation and constraining function of wrong beliefs in young children's understanding of deception. Cognition 1983;13:103-128.

21. Flobbe L, Verbrugge R, Hendriks P, Kr?mer I. Children's application of theory of mind in reasoning and language. J Logic Lang Inform 2008;17:417-442.

22. Jolliffe T, Baron-Cohen S. The strange stories test: A replication with high-functioning adults with autism or Asperger syndrome. J Autism Dev Disord 1999;29:395-406.

23. Baron-Cohen S, O'Riordan M, Jones R, Stone V, Plaisted K. A new test of social sensitivity: Detection of faux pas in normal children and children with Asperger syndrome. J Autism Dev Disord 1999;29:407-418.

24. Baron-Cohen S, Wheelwright S, Spong A, Scahill V, Lawson J. Are intuitive physics and intuitive psychology independent? A test with children with Asperger Syndrome. J Dev Learn Disord 2001;5:47-78.

25. Perner J, Lang B. Development of theory of mind and executive control. Trends Cogn Sci 1999;3:337-344.

26. Stone VE, Baron-Cohen S, Knight RT. Frontal lobe contributions to theory of mind. J Cogn Neurosci 1998;10:640-656.

27. Girli A, Tekin D. Investigating false belief levels of typically developed children and children with autism. Procedia Soc Behav Sci 2010;2: 1944-1950.

28. Corcoran R, Mercer G, Frith CD. Schizophrenia, symptomatology and social inference: investigating "theory of mind" in people with schizophrenia. Schizophr Res 1995;17:5-13.

29. Baron Cohen S, Jolliffe T, Mortimore C, Robertson M. Another advanced test of theory of mind: Evidence from very high functioning adults with autism or Asperger syndrome. J Child Psychol Psychiatry 1997;38:813-822.

30. Girli A. Psychometric properties of the Turkish child and adult form of "reading the mind in the eyes test". Psychology 2014;5:1321-1337.

31. Hughes C. Theory of mind grows up: reflections on new research on theory of mind in middle childhood and adolescence. J Experiment Child Psychol 2016;149:1-5.

32. Adachi T, Koeda T, Hirabayashi S, Maeoka Y, Shiota M, Wright EC, et al. The metaphor and sarcasm scenario test: A new instrument to help differentiate high functioning pervasive developmental disorder from attention deficit/hyperactivity disorder. Brain Dev 2004;26:301-306.

33. Baribeau DA, Doyle-Thomas KA, Dupuis A, Iaboni A, Crosbie J, Mc- 
Ginn H, et al. Examining and comparing social perception abilities across childhood-onset neurodevelopmental disorders. J Am Acad Child Adolesc Psychiatry 2015;54:479-486.

34. Demopoulos C, Hopkins J, Davis A. A comparison of social cognitive profiles in children with autism spectrum disorders and attention-deficit/hyperactivity disorder: a matter of quantitative but not qualitative difference? J Autism Dev Disord 2013;43:1157-1170.

35. Demurie E, De Corel M, Roeyers H. Empathic accuracy in adolescents with autism spectrum disorders and adolescents with attention-deficit/ hyperactivity disorder. Res Autism Spectr Disord 2011;5:126-134.

36. Downs A, Smith T. Emotional understanding, cooperation, and social behavior in high-functioning children with autism. J Autism Dev Disord 2004;34:625-635.

37. Dyck MJ, Ferguson K, Shochet IM. Do autism spectrum disorders differ from each other and from non-spectrum disorders on emotion recognition tests? Eur Child Adolesc Psychiatry 2001;10:105-116.

38. Sinzig J, Morsch D, Lehmkuhl G. Do hyperactivity, impulsivity and inattention have an impact on the ability of facial affect recognition in children with autism and ADHD? Eur Child Adolesc Psychiatry 2008;17:63-72.

39. Miranda A, Berenguer C, Rosell? B, Baixauli I, Colomer C. Social Cogni- tion in Children with High-Functioning Autism Spectrum Disorder and Attention-Deficit/Hyperactivity Disorder. Associations with Executive Functions. Front Psychol 2017;8:1035.

40. Lam KH, Ho CSH. Pragmatic skills in Chinese dyslexic children: Evidence from a parental checklist. Asia Pac J Dev Diff 2014;1:4-19.

41. Burden R. Is dyslexia necessarily associated with negative feelings of self worth? A review and implications for future research. Dyslexia 2008;14:188-196.

42. Cardillo R, Garcia RB, Mammarella IC, Cornoldi C. Pragmatics of language and theory of mind in children with dyslexia with associated language difficulties or nonverbal learning disabilities. Appl Neuropsychol Child 2018;7:245-256.

43. Buitelaar JK, Van der Wees M, Swaab-Barneveld H, Van der Gaag RJ. Theory of mind and emotion-recognition functioning in autistic spectrum disorders and in psychiatric control and normal children. Dev Psychopathol 1999;11:39-58.

44. Aspan N, Bozsik C, Gadoros J, Nagy P, Inantsy-Pap J, Vida P, et al. Emotion recognition pattern in adolescent boys with attention-deficit/ hyperactivity disorder. BioMed Res Int 2014;2014:761340.

45. Ahmed FS, Miller LS. Executive function mechanisms of theory of mind. J Autism Dev Disord 2011;41:667-678. 